\title{
Media Intervention
}

National Cancer Institute

\section{Source}

National Cancer Institute. Media Intervention. NCI Thesaurus. Code C15813.

An act or ministration that produces an effect to alter the course of a pathologic process

through the use of a variety of media, i.e., CD-ROM, Web application, video, telephone. 